\title{
Sufficient Subalgebras and the Relative Entropy of States of a von Neumann Algebra
}

\author{
Dénes Petz
}

Mathematisches Institut der Universität Tübingen, Auf der Morgenstelle 10, D-7400 Tübingen, Federal Republic of Germany

\begin{abstract}
A subalgebra $M_{0}$ of a von Neumann algebra $M$ is called weakly sufficient with respect to a pair $(\phi, \omega)$ of states if the relative entropy of $\phi$ and $\omega$ coincides with the relative entropy of their restrictions to $M_{0}$. The main result says that $M_{0}$ is weakly sufficient for $(\phi, \omega)$ if and only if $M_{0}$ contains the RadonNikodym cocycle $[D \phi, D \omega]_{t}$. Other conditions are formulated in terms of generalized conditional expectations and the relative Hamiltonian.
\end{abstract}

\section{Introduction}

Suppose that an experiment is described by a measurable space $(X, \mathscr{S})$ and the outcome of the experiment is governed by a probability measure $\mu$ on $\mathscr{S}$. In mathematical statistics the probability distribution $\mu$ belongs to a family $\Theta$ of probability distributions. A sub- $\sigma$-algebra $\mathscr{R}$ of $\mathscr{S}$ can be considered as application of a statistics or an indirect observation. $\mathscr{R}$ is defined to be sufficient with respect to the family $\Theta$ if the conditional distribution of $\mu \in \Theta$ does not depend on $\mu$. More precisely, for every $S \in \mathscr{S}$ there exist an $\mathscr{R}$-measurable function $\xi_{S}$ such that $\int_{R} \xi_{S} d \mu$ $=\mu(R \cap S)$ for every $R \in \mathscr{R}$ and $\mu \in \Theta$ (see [12 or 7, 28]). In mathematical statistics the case $|\Theta|=2$ is called the discrimination between two statistical hypotheses.

Let $\Theta=\{\mu, v\}$, and assume that $\mu, v \ll \lambda$. Halmos and Savage ([12]) proved that $\mathscr{R}$ is sufficient for $\{\mu, v\}$ if and only if the function

$$
\frac{d \mu}{d \lambda} / \frac{d v}{d \lambda}
$$

is $\mathscr{R}$-measurable. Another equivalent formulation due to Kullback and Leibler $[18]$ is based on the relative entropy of measures. Namely, $\mathscr{R}$ is sufficient if and only if

$$
S(\mu, v)=S\left(\mu_{0}, v_{0}\right),
$$

Permanent address: Mathematical Institute of HAS, Reáltanoda u. 13-15, H-1364 Budapest, P.O. Box. 127, Hungary 
where $S(\mu, v)\left(S\left(\mu_{0}, v_{0}\right)\right)$ denotes the relative entropy of $\mu$ and $v$ (that of their restrictions to $\mathscr{R})$.

The main goal of this paper is to investigate the equivalencies above in the noncommutative case. In the algebraic approach to quantum mechanics [10] a physical system corresponds to an operator algebra $M$, and a subalgebra $M_{0}$ of $M$ is selected for experimental observation. The determination through the coarse grained algebra $M_{0}$ provides only partial information about the state of the system. Mathematically, instead of a state (that is, a positive functional) of $M$ its restriction to $M_{0}$ is at our disposal (cf. [31]). Discussions concerning sufficiency of a subalgebra have been made first by Umegaki ([30], see also [11, 15]). The subalgebra $M_{0}$ is sufficient with respect to the states $\phi$ and $\omega$ of $M$, provided there is a conditional expectation $E$ (in the sense of Umegaki, see [30]) onto $M_{0}$ preserving $\phi$ and $\omega$, i.e. $\phi \circ E=\phi$ and $\omega \circ E=\omega$. By Takesaki's theorem ([29], see also $[27,10.1])$ projection of norm one onto a subalgebra preserving a given state is an infrequent object in the theory of operator algebras. Hence, the definition above is rather restrictive. On the other hand, in the present shape of quantum probability there exists no satisfactory mathematical formalism for conditioning. Therefore we try to involve the notion of relative entropy of states in the study of sufficiency instead of considering conditional expectations.

The relative entropy in an operator algebra setup was first studied by Umegaki [30] and it has provided a useful tool for mathematical physics in the papers of Lindblad [20, 21]. Araki [4] extended the notion of relative entropy to the case of normal states of arbitrary von Neumann algebra and its relation to the sufficiency problem was discussed also by Hiai et al. [13, 14].

In [23] we used the following definition. The subalgebra $M_{0}$ of $M$ is weakly sufficient for the pair $(\phi, \omega)$ if the relative entropy of $\phi$ and $\omega$ coincides with the relative entropy of their restrictions to $M_{0}$. Applying a method of Holevo [15] we characterized weakly sufficient commutative subalgebras of finite dimensional operator algebras. In the present paper we drop the assumption on commutativity and use a different method based on quasi-entropies (see [23, 24]. We prove that $M_{0}$ is weakly sufficient for $(\phi, \omega)$ if and only if the Radon-Nikodym cocycle $[D \phi, D \omega]_{t}$ is in $M_{0}$. This is equivalent to the condition $E_{\phi}=E_{\omega}$, where $E_{\psi}$ denotes the $\psi$-conditional expectation of Accardi and Cecchini [1] with respect to a state $\Psi$.

\section{Preliminaries}

Let $M$ be a von Neumann algebra with a faithful normal state $\omega$. We can assume that $M$ acts on a Hilbert space $H$ and $\omega$ is determined by a cyclic separating vector $\Omega$. If $\phi$ is another normal state, then the densely defined quadratic form $a \Omega \rightarrow \phi\left(a a^{*}\right)(a \in M)$ is closable and there exists an associated positive selfadjoint operator $\Delta$. It is characterized by the following properties. $M \Omega$ is a core for $\Delta^{1 / 2}$ and $\left\|\Delta^{1 / 2} a \Omega\right\|^{2}=\phi\left(a a^{*}\right)[17$, VI, Sect. 2]. $\Delta$ was called by Araki the relative modular operator of $\phi$ and $\omega$ and it is usually denoted by $\Delta(\phi, \omega)$ (see $[4,5])$.

The modular group of $\omega$ is a one-parameter group of automorphisms of $M$ and it looks like

$$
\sigma_{t}^{\omega}(a)=\Delta(\omega, \omega)^{i t} a \Delta(\omega, \omega)^{-i t}
$$


Another Radon-Nikodym derivative-like object for comparison of two states is the Radon-Nikodym cocycle discovered by Connes ([6], see also [5]). If $\phi$ is a faithful normal semifinite weight, then $[D \phi, D \omega]_{t}=u_{t}$ is a $\sigma^{\omega}$-cocycle and $\sigma_{t}^{\phi}=u_{t} \sigma_{t}^{\omega} u_{t}^{*}$. The formula $[D \phi, D \omega]_{t}=\Delta(\phi, \omega)^{i t} \Delta(\omega, \omega)^{-i t}$ forms a bridge between the two objects (see [5]).

The inner perturbation of a state was studied by Araki [2]. If $h=h^{*} \in M$, then there is a state $\omega^{h}$ determined by

$$
\left[D \omega^{h}, D \omega\right]_{t}=e^{i t(H+h)} e^{-i t H},
$$

where $H$ stands for $\log \Delta(\omega, \omega)$. The main properties of the perturbed state are summarized in [3].

Let $f: \mathbb{R}^{+} \rightarrow \mathbb{R}$ be a continuous function. The quasi-entropy $S_{f}(\phi, \omega)$ is defined as $\langle f(\Delta(\phi, \omega)) \Omega, \Omega\rangle$. It possesses nice convexity properties when $f$ is operator convex (see $[23,24])$. The choice $f(t)=-\log t$ gives the relative entropy:

$$
S(\phi, \omega)=-\langle\log \Delta(\phi, \omega) \Omega, \Omega\rangle .
$$

Monotonicity of quasi-entropies means the following assertion. Assume that $M_{0}$ and $M$ are von Neumann algebras and $\alpha: M_{0} \rightarrow M$ is a 2-positive unit preserving mapping. If $\phi$ and $\omega$ are faithful normal states on $M$, then $S_{f}(\phi \circ \alpha, \omega \circ \alpha) \leqq S_{f}(\phi, \omega)$, provided that $-f$ is operator monotone ([24]).

If $M_{0}$ is a subalgebra of $M$, then $\omega$-preserving conditional expectation of $M$ onto $M_{0}$ does not exists always. However, there exists a very natural completely positive mapping $E_{\omega}: M \rightarrow M_{0}$, which is defined by Accardi and Cecchini ([1]) and may be called generalized conditional expectation for several reasons. The $\omega$-conditional expectation $E_{\omega}$ has a convenient description using standard forms [22]. Assume that $M\left(M_{0}\right)$ has the standard form $(M, H, J, \mathscr{P})\left(\left(M_{0}, H_{0}, J_{0}, \mathscr{P}_{0}\right)\right)$, and let $l: M_{0} \rightarrow M$ be the embedding. If $\Omega \in \mathscr{P}\left(\Omega_{0} \in \mathscr{P}_{0}\right)$ is the vector representative for the state $\omega\left(\omega\right.$ restricted to $\left.M_{0}\right)$ then

$$
V_{\omega} a \Omega_{0}=l(a) \Omega \quad\left(a \in M_{0}\right)
$$

defines a contraction of $H_{0}$ into $H$. The $\omega$-conditional expectation is defined as

$$
E_{\omega}(b)=J_{0} V_{\omega}^{*} J b J V_{\omega} J_{0} \quad(b \in M) .
$$

Concerning the $\omega$-conditional expectation we refer to the papers [1] and [20].

\section{Main Result}

Let $M$ be a von Neumann algebra with faithful normal states $\phi$ and $\omega$. When $M_{0}$ is a von Neumann subalgebra of $M$, then $\phi_{0}$ and $\omega_{0}$ stand for the the restriction of $\phi$ and $\omega$, respectively. By the monotonicity property of the relative entropy, we have $S\left(\phi_{0}, \omega_{0}\right) \leqq S(\phi, \omega)$. We say that $M_{0}$ is weakly sufficient with respect to the pair $(\phi, \omega)$ if $S\left(\phi_{0}, \omega_{0}\right)=S(\phi, \omega)$ (see [23]). Recall that $M_{0}$ is sufficient if there exists a conditional expectation $E: M \rightarrow M_{0}$ such that $\phi \circ E=\phi$ and $\omega \circ E=\omega$. In particular, sufficiency implies weak sufficiency.

In the rest of this section we assume that $M$ acts on a Hilbert space $H$ and $\omega$ is given by the cyclic and separating vector $\Omega \in H$. We consider the natural 
representation of $M_{0}$ on $H_{0}=\left[M_{0} \Omega\right]$, and $P$ denotes the orthogonal projection of $H$ onto $H_{0}$. We shall shorten our notation to $\Delta\left(\Delta_{0}\right)$ for the relative modular operator $\Delta(\phi, \omega)\left(\Delta\left(\phi_{0}, \omega_{0}\right)\right)$.

Lemma 1. Assume that $M_{0}$ is weakly sufficient for $(\phi, \omega)$ and $S(\phi, \omega)$ is finite. Then

$$
S_{f_{t}}(\phi, \omega)=S_{f_{t}}\left(\phi_{0}, \omega_{0}\right)
$$

where $f_{t}(\lambda)=1 / \lambda+t(0<t<\infty)$.

Proof. Using the formula

$$
\log x=\int_{0}^{\infty}(1+t)^{-1}-(x+t)^{-1} d t
$$

we write

$$
\begin{aligned}
S(\phi, \omega) & =-\langle\log \Delta \Omega, \Omega\rangle=\int_{0}^{\infty}\left\langle(\Delta+t)^{-1} \Omega, \Omega\right\rangle-(1+t)^{-1} d t \\
& =\int_{0}^{\infty} S_{f_{t}}(\phi, \omega)-(1+t)^{-1} d t
\end{aligned}
$$

Since $-f_{t}$ is operator monotone, by the monotonicity of the quasi-entropies, we have $S_{f_{t}}\left(\phi_{0}, \omega_{0}\right) \leqq S_{f_{t}}(\phi, \omega)$ and $S(\phi, \omega)=S\left(\phi_{0}, \omega_{0}\right)$ implies that $S_{f_{t}}(\phi, \omega)$ $=S_{f_{t}}\left(\phi_{0}, \omega_{0}\right)$ for almost all $t \in \mathbb{R}^{+}$. By continuity we obtain the equality for all $t \in \mathbb{R}^{+}$.

Lemma 2. For $\lambda>0$, we have

$$
P\left(\lambda+P \Delta_{0} P\right)^{-1} P \leqq P(\lambda+\Delta)^{-1} P .
$$

Proof. $\Delta$ is the associated positive selfadjoint operator to the densely defined closable quadratic form $q: a \Omega \rightarrow \phi\left(a a^{*}\right),(a \in M)$ and $\Delta_{0}$ is associated to the restriction $q \mid M_{0} \Omega$. Let $\int_{0}^{\infty} \lambda d E(\lambda)$ be the spectral decomposition of $\Delta$, and define $H_{n}=\int_{0}^{n} \lambda d E(\lambda)$. Then $\left(\lambda+H_{n}\right)^{-1} \rightarrow(\lambda+\Delta)^{-1}$ strongly for all $\lambda>0$.

The set $\left\{a \Omega+\eta: \eta \in H, P \eta=0, a \in M_{0}\right\}$ is a core for $\left(P \Delta_{0} P\right)^{1 / 2}=P \Delta_{0}^{1 / 2} P$. Evidently,

$$
\begin{gathered}
\left\|\left(P \Delta_{0} P\right)^{1 / 2}(a \Omega+\eta)\right\|^{2}=\left\|\Delta_{0}^{1 / 2} a \Omega\right\|^{2}=\phi\left(a a^{*}\right)=\left\|\Delta^{1 / 2} a \Omega\right\|^{2} \\
\geqq\left\|\left(P H_{n} P\right)^{1 / 2}(a \Omega+\eta)\right\|^{2},
\end{gathered}
$$

and we establish $P H_{n} P \leqq P \Delta_{0} P$. Then

Since

$$
\left(\lambda+P \Delta_{0} P\right)^{-1} \leqq\left(\lambda+P H_{n} P\right)^{-1} .
$$

$$
P\left(\lambda+P H_{n} P\right)^{-1} P \leqq P\left(\lambda+H_{n}\right)^{-1} P
$$

(see [8]) we arrive at

$$
P\left(\lambda+P \Delta_{0} P\right)^{-1} P \leqq P\left(\lambda+H_{n}\right)^{-1} P,
$$

and letting $n \rightarrow \infty$, we complete the proof. 
Lemma 3. Assume that

$$
\left\langle\left(\lambda+P \Delta_{0} P\right)^{-1} \Omega, \Omega\right\rangle=\left\langle(\lambda+\Delta)^{-1} \Omega, \Omega\right\rangle
$$

for all $\lambda>0$. Then $f(\Delta) \Omega=f\left(\Delta_{0}\right) \Omega$ for every bounded continuous function $f: \mathbb{R}^{+} \rightarrow \mathbb{C}$.

Proof. Using Lemma 2 we can estimate

$$
\begin{aligned}
& \left\|P(\lambda+\Delta)^{-1} \Omega-\left(\lambda+\Delta_{0}\right)^{-1} \Omega\right\|^{2}=\left\|\left(P(\lambda+\Delta)^{-1} P-P\left(\lambda+P \Delta_{0} P\right)^{-1} P\right) \Omega\right\|^{2} \\
& \leqq\left\|\left(P(\lambda+\Delta)^{-1} P-P\left(\lambda+P \Delta_{0} P\right)^{-1} P\right)^{1 / 2}\right\|^{2}\left\langle P(\lambda+\Delta)^{-1} \Omega-\left(\lambda+\Delta_{0}\right)^{-1} \Omega, \Omega\right\rangle \text {, }
\end{aligned}
$$

whenever $\lambda>0$. By the hypothesis, we have $P(\lambda+\Delta)^{-1} \Omega=\left(\lambda+\Delta_{0}\right)^{-1} \Omega$ for all $\lambda>0$, and through analytic continuation for all $\lambda \in \mathbb{C}-\mathbb{R}^{-}$. Derivating by $\lambda$ we obtain $P(\lambda+\Delta)^{-2} \Omega=\left(\lambda+\Delta_{0}\right)^{-2} \Omega$. Hence

$$
\begin{aligned}
\left\|(\lambda+\Delta)^{-1} \Omega\right\|^{2} & =\left\langle(\lambda+\Delta)^{-2} \Omega, \Omega\right\rangle=\left\langle\left(\lambda+\Delta_{0}\right)^{-2} \Omega, \Omega\right\rangle \\
& =\left\langle P(\lambda+\Delta)^{-1} \Omega,(\lambda+\Delta)^{-1} \Omega\right\rangle,
\end{aligned}
$$

and $(\lambda+\Delta)^{-1} \Omega$ must be in $H_{0}$. So $(\lambda+\Delta)^{-1} \Omega=\left(\lambda+\Delta_{0}\right)^{-1} \Omega,\left(\lambda \in \mathbb{C}-\mathbb{R}^{-}\right)$. Looking at the formula for spectral resolution [9, p. 1202], we can establish $E((s, t), \Delta) \Omega=E\left((s, t), \Delta_{0}\right) \Omega$ for every open interval $(s, t) \subset \mathbb{R}$. Reference to the spectral theorem makes the proof complete.

Theorem 4. Assume that $S(\phi, \omega)$ is finite. For a subalgebra $M_{0}$ of $M$ the following conditions are equivalent.

(i) $[D \phi, D \omega]_{t} \in M_{0}$ for every $t \in \mathbb{R}$.

(ii) There exists a subalgebra of $M_{0}$ which is sufficient for $(\phi, \omega)$.

(iii) $M_{0}$ is weakly sufficient for $(\phi, \omega)$.

(iv) $[D \phi, D \omega]_{t}=\left[D \phi_{0}, D \omega_{0}\right]_{t}$ for every $t \in \mathbb{R}$.

(v) $E_{\phi}=E_{\omega}$.

(vi) $\phi \circ E_{\omega}=\phi$.

(vii) $\omega \circ E_{\phi}=\omega$.

Proof. Suppose (i) and let $N$ be the subalgebra generated by $\left\{[D \phi, D \omega]_{t}: t \in \mathbb{R}\right\}$. Then $N$ is stable under the modular group of $\omega$ and there is a conditional expectation $E: M \rightarrow N$ preserving $\omega$. The converse of Connes' theorem $[6,27,5.1]$ guarantees a weight $\phi^{\prime}$ on $N$ such that $[D \phi, D \omega]_{t}=\left[D \phi^{\prime}, D(\omega \mid N)\right]_{t}$. On the other hand $\left[D\left(\phi^{\prime} \circ E\right), D \omega\right]_{t}=\left[D \phi^{\prime}, D(\omega \mid N)\right]_{t}$ (see $\left.[27,10.5]\right)$ and $\phi^{\prime} \circ E$ must be $\phi$. Consequently, $E$ preserves also $\phi$.

(ii) implies (iii) according to the monotonicity theorem.

To prove (iii) $\rightarrow$ (iv), we first note that

$$
[D \phi, D \omega]_{t} \Omega=\Delta(\phi, \omega)^{i t} \Delta(\omega, \omega)^{-i t} \Omega=\Delta^{i t} \Omega,
$$

and similarly $\left[D \phi_{0}, D \omega_{0}\right]_{t} \Omega=\Delta_{0}^{i t} \Omega$. Due to Lemma $3 \quad[D \phi, D \omega]_{t} \Omega$ $=\left[D \phi_{0}, D \omega_{0}\right]_{t} \Omega$, and we conclude (iv).

In order to prove (iv) $\rightarrow(\mathrm{v})$, let $(M, H, J, \mathscr{P})\left(\left(M_{0}, H_{0}, J_{0}, \mathscr{P}_{0}\right)\right)$ be the standard form of $M$ and $M_{0}$, respectively. Define the contractions $V_{\omega}, V_{\phi}: H_{0} \rightarrow H$ by the formulas

$$
V_{\omega} a \Omega=\imath(a) \Omega \quad \text { and } \quad V_{\phi} a \Phi_{0}=\imath(a) \Phi \quad\left(a \in M_{0}\right)
$$


where $\Omega \in \mathscr{P}$ and $\Phi \in \mathscr{P}\left(\Omega \in \mathscr{P}_{0}\right.$ and $\left.\Phi_{0} \in \mathscr{P}_{0}\right)$ are the vector representatives for the states $\omega$ and $\phi\left(\omega_{0}\right.$ and $\left.\phi_{0}\right)$, moreover $\imath: M_{0} \rightarrow M$ is the inclusion. By assumption

$$
V_{\omega}\left(a\left[D \phi_{0}, D \omega_{0}\right]_{t} \Omega\right)=\imath(a)[D \phi, D \omega]_{t} \Omega
$$

for all $a \in M_{0}$ and $t \in \mathbb{R}$. Since $\left[D \phi_{0}, D \omega_{0}\right]_{t} \Omega=\Delta\left(\phi_{0}, \omega_{0}\right)^{i t} \Omega$, and $\Omega \in D\left(\Delta\left(\phi_{0}, \omega_{0}\right)^{1 / 2}\right)$ [in fact, $\Delta\left(\phi_{0}, \omega_{0}\right)^{1 / 2} \Omega=\Phi_{0}$ ] the function

$$
F_{a}(t): t \rightarrow V_{\omega}\left(a\left[D \phi_{0}, D \omega_{0}\right]_{t} \Omega\right)
$$

has continuous extension to the strip $S=\{z \in \mathbb{C}:-1 / 2 \leqq \operatorname{Im} z \leqq 0\}$, which is analytic on $S^{0}=\{z \in \mathbb{C}:-1 / 2<\operatorname{Im} z<0\} \quad[26,9.15]$. Similarly, $G_{a}(z)$ $=a \Delta\left(\phi_{0}, \omega_{0}\right)^{i t} \Omega$ is continuous on $S$ and analytic on $S^{0}$. Since $F_{a}(t)=G_{a}(t)$ for every $t \in \mathbb{R}$, we can conclude

$$
V_{\omega}\left(a \Phi_{0}\right)=F_{a}(-i / 2)=G_{a}(-i / 2)=a \Phi \quad\left(a \in M_{0}\right),
$$

and this means that $V_{\phi}=V_{\omega}$. Therefore, $E_{\phi}=E_{\omega}$.

The implications (v) $\rightarrow$ (vi) and (v) $\rightarrow$ (vii) are obvious and both (vi) and (vii) imply (iii) by the monotonicity theorem. Finally, (iv) $\rightarrow$ (i) is trivial.

It seems worth noting that weak sufficiency for a pair $(\phi, \omega)$ is not symmetrical by definition, but it turns out to be such according to Theorem 4 . The subalgebra generated by $\left\{[D \phi, D \omega]_{t}: t \in \mathbb{R}\right\}$ is the smallest weakly sufficient subalgebra and it is sufficient.

Corollary 5. If $S(\phi, \omega)$ is finite, $M_{0}$ is weakly sufficient for $(\phi, \omega)$ and there is a conditional expectation of $M$ onto $M_{0}$ which preserves $\phi$ or $\omega$, then $M_{0}$ is sufficient.

Proof. If, for example, $\phi \circ E=\phi$, then $E=E_{\phi}$ must hold, and according to condition (viii) in Theorem $4 \omega \circ E=\omega$.

Theorem 6. Let $M_{0}$ be a von Neumann subalgebra of the von Neumann algebra $M$. Assume that $\omega$ is a faithful normal state on $M$ and denote by $\omega^{h}$ the perturbation of $\omega$ by $h=h^{*} \in M$. Then the following conditions are equivalent.

(i) $\sigma_{s}^{\omega}\left(\left[D \omega^{h}, D \omega\right]_{t}\right) \in M_{0}(s, t \in \mathbb{R})$.

(ii) $\sigma_{s}^{\omega}(h) \in M_{0}(s \in \mathbb{R})$.

(iii) $E_{\omega}(h)=h$, where $E_{\omega}$ denotes the $\omega$-conditional expectation of $M$ into $M_{0}$.

Proof. We abbreviate $\log \Delta(\omega, \omega)$ as $H$. By straightforward computation, we have for $\xi \in D(H)$,

$$
\lim \left(e^{i t(H+h)} e^{-i t H} \xi-\xi\right) / i t=h \xi
$$

as $t \rightarrow 0$. We show that

$$
\left(e^{i t(H+h)} e^{-i t H}-I\right) / t=\left(e^{i t(H+h)}-e^{i t H}\right) e^{-i t H} / t
$$

is bounded in a neighbourhood of 0 . We need the norm convergent expansion

$$
e^{i t(H+h)}=\sum_{n=0}^{\infty} U_{n}(t)
$$

where $U_{0}(t)=e^{i t H}, U_{n+1}(t)=-i \int_{0}^{t} e^{i(t-s) H} h U_{n}(s) d s$ and the estimate

$$
\left\|U_{n}(t)\right\| \leqq t^{n}\|h\|^{n} / n !
$$


(see [17, IX, Sect. 2]). So

$$
\left\|\left(e^{i t(H+h)}-e^{i t H}\right) / t\right\|=\left\|\sum_{n=1}^{\infty} U_{n}(t) / t\right\| \leqq \sum_{n=1}^{\infty} t^{n-1}\|h\|^{n} / n !=\left(e^{t\|h\|}-1\right) / t
$$

is a bounded function of $t$ in a neighbourhood of 0 . Consequently, the limit above exists for every $\xi \in H$.

Now we prove (i) $\rightarrow$ (ii). Since

$$
\sigma_{s}^{\omega}\left(\left[D \omega^{h}, D \omega\right]_{t}\right)-I=e^{i s H}\left(e^{i t(H+h)} e^{-i t h}-I\right) e^{-i s H}
$$

by the previous considerations, it follows that the strong derivative of $\sigma_{s}^{\omega}\left(\left[D \omega^{h}, D \omega\right]_{t}\right)$ at $t=0$ is $-i \sigma_{s}^{\omega}(h)$.

Conversely, we refer to the expansion

$$
\left[D \omega^{h}, D \omega\right]_{t}=\sum_{n=0}^{\infty} i^{n} \int_{0}^{t} d t_{1} \int_{0}^{t_{1}} d t_{2} \ldots \int_{0}^{t_{n}-1} d t_{n} \sigma_{t_{n}}^{\omega}(h) \ldots \sigma_{t_{1}}^{\omega}(h)
$$

(see [2]) which shows immediately the implication (ii) $\rightarrow$ (i).

The equivalence (ii) $\leftrightarrow$ (iii) is in Theorem 5.1 of [1].

Corollary. The subalgebra $M_{0}$ is is weakly sufficient for the pair $\left(\omega^{h}, \omega\right)$ (where $\omega^{h}$ is an inner perturbation of $\omega$ ) if and only if $h$ is a fixed point of the $\omega$-conditional expectation of $M$ into $M_{0}$.

\section{Discussions}

Let $M$ be a von Neumann algebra with faithful normal states $\phi$ and $\omega$. In [25] Raggio introduced a transition probability $P_{A}(\phi, \omega)$ which turned out to be a quasi-entropy by the function $f(t)=t^{1 / 2}$ (see [23]). Suppose that $M_{0}$ is a subalgebra of $M$. The equality $P_{A}(\phi, \omega)=P_{A}\left(\phi\left|M_{0}, \omega\right| M_{0}\right)$ can be studied by our methods, and it is equivalent to the conditions (i)-(vii) in Theorem 4.

The outstanding problem of joint convexity of the relative entropy functional was proved by Lieb [19]. We recall the argument in [24] deducing convexity from monotonicity. If $\phi$ and $\omega$ normal states on $M(i=1,2)$, then consider the following states on $N=M \oplus M$ :

$$
\phi_{12}=\lambda \phi_{1}+(1-\lambda) \phi_{2} \quad \text { and } \quad \omega_{12}=\lambda \omega_{1}+(1-\lambda) \omega_{2} .
$$

So

$$
S\left(\phi_{12}, \omega_{12}\right)=\lambda S\left(\phi_{1}, \omega_{1}\right)+(1-\lambda) S\left(\phi_{2}, \omega_{2}\right) .
$$

On the other hand,

$$
S\left(\phi_{12}\left|N_{0}, \omega_{12}\right| N_{0}\right)=S\left(\lambda \phi_{1}+(1-\lambda) \phi_{2}, \lambda \omega_{1}+(1-\lambda) \omega_{2}\right),
$$

where $N_{0}=\{a \oplus a: a \in M\}$ is a subalgebra of $N$. The monotonicity yields

$$
S\left(\lambda \phi_{1}+(1-\lambda) \phi_{2}, \lambda \omega_{1}+(1-\lambda) \omega_{2}\right) \leqq \lambda S\left(\phi_{1}, \omega_{1}\right)+(1-\lambda) S\left(\phi_{2}, \omega_{2}\right) .
$$

We are going to find a condition for the equality. By Theorem 4 the equality holds if $\left[D \phi_{12}, D \omega_{12}\right]_{t} \in N_{0}$ for $t \in \mathbb{R}$. Since

$$
\left[D \phi_{12}, D \omega_{12}\right]_{t}=\left[D \phi_{1}, D \omega_{1}\right]_{t} \oplus\left[D \phi_{2}, D \omega_{2}\right]_{t},
$$


the equality $\left[D \phi_{1}, D \omega_{1}\right]_{t}=\left[D \phi_{2}, D \omega_{2}\right]_{t}$ is a necessary and sufficient condition. In terms of densities this reads

$$
\varrho_{1}^{-i t} \varrho_{2}^{i t}=v_{1}^{-i t} v_{2}^{i t},
$$

where $v_{i}$ and $\varrho_{i}$ are the densities of $\phi_{i}$ and $\omega_{i}$, respectively $(i=1,2)$.

Acknowledgements. This work was done while the author stayed at the Mathematical Institute of the University Tübingen supported by a Forschungsstipendium from the Alexander von Humboldt-Stiftung. It is a pleasure to thank W. Arendt, I. Csiszár, B. Kümmerer, G. Raggio, and L. Zsidó for valuable conversations. The author is grateful to the referee for his suggestions.

\section{References}

1. Accardi, L., Cecchini, C.: Conditional expectation in von Neumann algebras and a theorem of Takesaki. J. Funct. Anal. 45, 245-273 (1982)

2. Araki, H.: Relative Hamiltonian for faithful normal states of a von Neumann algebra. Publ. Res. Inst. Math. Sci. 9, 165-209 (1973)

3. Araki, H.: Recent developments in the theory of operator algebras and their significance in theoretical physics. Symposia Math., XX, 395-424. New York: Academic Press 1976

4. Araki, H.: Relative entropy of states of von Neumann algebras. I, II. Publ. Res. Inst. Math. Sci. 11, 809-833 (1976) and 13, 173-192 (1977)

5. Araki, H., Masuda, T.: Positive connes and $L_{p}$-spaces for von Neumann algebras. Publ. Res. Inst. Math. Sci. 18, 339-411 (1982)

6. Connes, A.: Une classification des facteurs de type III. Ann. Sci. Ec. Norm. Supér. 6, 133-252 (1973)

7. Csiszár, I.: Information type measure of difference of probability distributions and indirect observations. Stud. Sci. Math. Hung. 2, 299-318 (1967)

8. Davis, C.: A Schwarz inequality for convex operator functions. Proc. Am. Math. Soc. 8, 42-44 (1957)

9. Dunford, N., Schwartz, J.T.: Linear operators. Part II. New York: Interscience 1958

10. Emch, G.G.: Algebraic methods in statistical mechanics and quantum field theory. New York: Wiley 1972

11. Gudder, S., Merchand, J.P.: Noncommutative probability on von Neumann algebras. J. Math. Phys. 13, 799-806 (1972)

12. Halmos, P.R., Savage, L.J.: Application of the Radon-Nikodym theorem to the theory of sufficient statistics. Ann. Math. Stat. 20, 225-241 (1949)

13. Hiai, F., Ohya, M., Tsukada, M.: Sufficiency, KMS condition and relative entropy in von Neumann algebras. Pac. J. Math. 96, 99-109 (1981)

14. Hiai, F., Ohya, M., Tsukada, M.: Sufficiency and relative entropy in *-algebras with applications in quantum systems. Pac. J. Math. 107, 117-140 (1983)

15. Holevo, A.S.: Some estimates for the amount of information transmittable by a quantum communication channel (in Russian). Probl. Peredachi Inf. 9, 3-11 (1973)

16. Holevo, A.S.: Investigations in the general theory of statistical decisions. Am. Math. Soc. Proc. Steklov Inst. Math. 124 (1978)

17. Kato, T.: Perturbation theory for linear operators. Berlin, Heidelberg, New York: Springer 1966

18. Kullback, S., Leibler, R.A.: On information and sufficiency. Ann. Math. Stat. 22, 79-86 (1951)

19. Lieb, E.H.: Convex trace functions and the Wigner-Yanase-Dyson conjecture. Adv. Math. 11, 267-288 (1973)

20. Lindblad, G.: Entropy, information and quantum measurement. Commun. Math. Phys. 33, 305-322 (1973)

21. Lindblad, G.: Expectation and entropy inequalities for finite quantum systems. Commun. Math. Phys. 39, 111-119 (1974) 
22. Petz, D.: A dual in von Neumann algebras with weights. Q. J. Math. Oxf. 35, 475-483 (1984)

23. Petz, D.: Properties of quantum entropy, quantum probability and applications II. Accardi, L., von Waldenfels, W. (eds.). Lecture Notes in Mathematics. Vol. 1136, pp. 428-441. Berlin, Heidelberg, New York: Springer 1985

24. Petz, D.: Quasi-entropies for states of a von Neumann algebra. Publ. Res. Inst. Math. Sci. 21, 787-800 (1985)

25. Raggio, G.A.: Comparison of Uhlmann's transition probability with the one induced by the natural positive cone of a von Neumann algebra in standard form. Lett. Math. Phys. 6, 233-236 (1982)

26. Strătilă, S., Zsidó, L.: Lectures on von Neumann algebras. Tunbridge Wells: Abacuss Press 1979

27. Strătilă, S.: Modular theory of operator algebras. Tunbridge Wells: Abacus Press 1981

28. Strasser, H.: Mathematical theory of statistics. Berlin: de Gruyter 1985

29. Takesaki, M.: Conditional expectations in von Neumann algebras. J. Funct. Anal. 9, 306-321 (1972)

30. Umegaki, H.: Conditional expectations in an operator algebra IV (entropy and information). Kodai Math. Sem. Rep. 14, 59-85 (1962)

31. Wolfe, J.C., Emch, G.G.: $C^{*}$-algebraic formalism for coarse graining. I. General theory. J. Math. Phys. 15, 1343-1347 (1974)

Communicated by H. Araki

Received November 28, 1985; in revised form January 23, 1986 
